\title{
负折射率薄膜热辐射的 TM偏振特性研究
}

\author{
崔炎, 黄勇 ${ }^{*}$ 李雯, 王浚
}

北京航空航天大学航空科学与工程学院, 北京 100191

* 联系人, E-mail: huangy@buaa.edu.cn

2008-10-15 收稿, 2008-11-25 接受

国家自然科学基金(批准号: 50606003)、航空科学基金(编号: 2007ZA51006)资助项目

摘要 利用传输矩阵方法及基尔霍夫定律推导了 $\mathrm{TM}$ 偏振波的偏振特性计算公式. 在研究 $\mathrm{TM}$ 波特 性的同时, 评估了体系参数对薄膜辐射方向性及相干性的影响, 以及修逝波对 TM 偏振波偏振特性 的影响. 结果表明负折射率薄膜的热辐射特性是由两个作用共同影响所产生的结果, 一个作用是薄 膜结构对电磁波的干涉效应, 另一个作用是负折射率材料对修逝波的放大导致的光子隧道效应. 通 过调整薄膜结构的体系参数, 可以对其热辐射的方向特征和光谱特征进行相应的调制.

关键词

热辐射 负折射率

薄膜

修逝波

发射率
1968 年, 前苏联物理学家Veselago首次提出了负 折射率材料的概念 $[1] .20$ 世纪 90 年代后半期, 由于英 国人Pendry的理论研究, 负介电常数和负磁导率材料 的真实性才从理论上得到了证明. 在Pendry研究的基 础上, Shelby等人 ${ }^{[2]}$ 以铜作为主要的复合材料, 成功 地研制出负折射率材料并且验证了微波段负折射现 象. 从此, 负折射率材料作为一种崭新的人工复合材 料逐渐成为了众多学者关注的焦点 ${ }^{[3 \sim 5]}$.

负折射率材料具有诸多奇异的特性，例如产生 反常 Cerenkov效应、逆Doppler效应 ${ }^{[1]}$, 形成“完美透 镜” 和放大修逝波的功能 6$]$. 早期的负折射率材料研

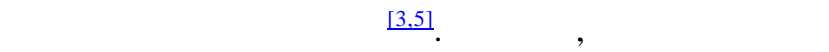
的发展以及新材料、新几何构型的发明, 红外波段甚 至是可见光段的负折射率材料也相继研制成功 ${ }^{[4,7]}$. 2008 年, 两种新的纳米级“超材料” 相继问世. 利用 这两种新材料, 加州大学伯克利分校的Zhang等人首 次实现了可见光波段的“负折射”现象，同时也第一 次成功研制出了真正具有光学磁性的材料 ${ }^{[8,9]}$. 这项 研究结果被誉为负折射率材料研究里程碑式的工作. 负折射率材料具备了控制光的特性, 从而使得负折 射率材料在隐身技术领域的应用研究成为了可能 ${ }^{[10]}$.

本文在波动光学和电动力学的基础上进行负折 射率薄膜偏振特性的理论研究, 利用传输矩阵法和 基尔霍夫定律建立起负折射率薄膜发射率与反射率、
透射率之间的关系. 同时还讨论了修逝波放大效应 对负折射率薄膜偏振特性的影响, 并分析了频率、角 度及薄膜厚度对薄膜发射、反射的影响.

\section{1 模型建立}

考虑如图 1 所示的物理模型, 假设厚度为 $d$ 的薄 膜置于真空环境中, 材料均匀、各向同性, 其相对介 电常数为 $\varepsilon$ 、相对磁导率为 $\mu$. 一束单色平面电磁波从 $z=0$ 处界面射入薄膜, 入射角为 $\theta$. 任何平面电磁波 都可以分解成振动方向垂直于入射面的 TE 波和振动

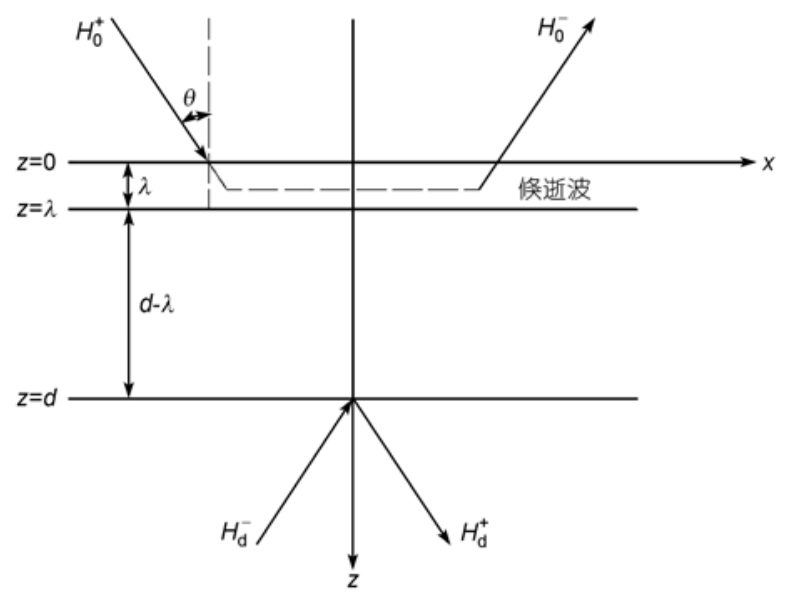

图 1 物理模型 
方向平行于入射面的 TM 波. 这两种波互相独立，因 此图 1 所示物理模型存在着 TE 和 TM 偏振两种模式. 本文研究其热辐射的 TM 偏振特性, 根据 Maxwell 方 程的对称性, TE 偏振有与 TM 偏振相似的特性.

对于 $\mathrm{TM}$ 偏振来说, 其磁矢量垂直于入射面 $x z$ 平面, 即磁矢量平行于 $y$ 轴. 如图 1 所示, 通过 $z=0$ 界面向内的电磁波的磁矢量为:

$$
H_{0}^{+}=A_{0} \exp \left[i \omega t-i\left(k_{x} x+k_{z} z\right)\right],
$$

通过 $z=0$ 界面向外的电磁波的磁矢量为:

$$
H_{0}^{-}=B_{0} \exp \left[i \omega t+i\left(k_{x} x-k_{z} z\right)\right],
$$

通过 $z=d$ 界面向外的电磁波的磁矢量为:

$$
H_{\mathrm{d}}^{+}=A_{\mathrm{d}} \exp \left[i \omega t-i k_{x} x-i k_{z}(z-d)\right],
$$

通过 $z=d$ 界面向内的电磁波的磁矢量为:

$$
H_{\mathrm{d}}^{-}=B_{\mathrm{d}} \exp \left[i \omega t+i k_{x} x-i k_{z}(z-d)\right]=0,
$$

式中 $\omega$ 为入射电磁波的角频率, $k_{x}, k_{z}$ 为波矢在 $x$ 和 $z$ 方向的分量; $A_{0}, A_{\mathrm{d}}, B_{0}$ 和 $B_{\mathrm{d}}$ 为各电磁波的幅值.

根据传输矩阵法 ${ }^{[11,12]}$, 利用电磁场的边值关系, 推导出薄膜各边界处电磁波幅值之间的关系为:

$$
\begin{aligned}
\left(\begin{array}{l}
A_{0} \\
B_{0}
\end{array}\right)= & M\left(\begin{array}{l}
A_{\mathrm{d}} \\
B_{\mathrm{d}}
\end{array}\right)=\left(\begin{array}{ll}
M_{11} & M_{12} \\
M_{21} & M_{22}
\end{array}\right)\left(\begin{array}{l}
A_{\mathrm{d}} \\
B_{\mathrm{d}}
\end{array}\right) \\
= & P_{1} D_{1}^{-1} D_{2} P_{2} D_{2}^{-1} D_{3}\left(\begin{array}{l}
A_{\mathrm{d}} \\
B_{\mathrm{d}}
\end{array}\right) \\
= & \left(\begin{array}{cc}
1 & 1 \\
k_{z} & -k_{z}
\end{array}\right)^{-1}\left(\begin{array}{cc}
1 & 1 \\
\frac{k_{z}^{\prime}}{\varepsilon} & -\frac{k_{z}^{\prime}}{\varepsilon}
\end{array}\right)\left(\begin{array}{cc}
e^{-i k_{z}^{\prime} d} & 0 \\
0 & e^{i k_{z}^{\prime} d}
\end{array}\right) \\
& \times\left(\begin{array}{cc}
1 & 1 \\
\frac{k_{z}^{\prime}}{\varepsilon} & -\frac{k_{z}^{\prime}}{\varepsilon}
\end{array}\right)^{-1}\left(\begin{array}{cc}
1 & 1 \\
k_{z} & -k_{z}
\end{array}\right)\left(\begin{array}{l}
A_{d} \\
B_{\mathrm{d}}
\end{array}\right),
\end{aligned}
$$

式中 $k_{z}^{\prime}$ 是薄膜传播的电磁波波矢在 $z$ 方向的分量, 根 据体系参量匹配的关系有 $k_{\mathrm{x}}^{\prime} \equiv k_{\mathrm{x}}=\omega n_{1} \sin \theta / c$, 所以 $k_{\mathrm{z}}^{\prime 2}+k_{\mathrm{x}}^{\prime 2}=\varepsilon \mu \omega^{2} / c^{2}$, 其中 $n_{1}=1$ 为真空的折射率. 对 于半无限大介质可知 $B_{\mathrm{d}}=0$, 因此可以得到薄膜的透 射因子 $t$ 和反射因子 $r$ 分别为:

$$
\begin{aligned}
& t=\frac{A_{d}}{A_{0}}=\frac{1}{M_{11}}, \\
& r=\frac{B_{0}}{A_{0}}=\frac{M_{21}}{M_{11}} .
\end{aligned}
$$

因此薄膜的透射率 $T$ 和反射率 $R$ 分别为:

$$
T=t t^{*}=\left|\frac{1}{M_{11}}\right|^{2},
$$

$$
R=r r^{*}=\left|\frac{M_{21}}{M_{11}}\right|^{2} .
$$

根据基尔霍夫辐射定律, 热平衡条件下薄膜的 单色定向发射率等于它的单色定向吸收率, 所以可 以得到:

$$
E=1-T-R .
$$

\section{2 负折射率薄膜参数的确定与计算}

负折射率材料在红外及可见光等高频段有重要 的应用前景, 其特性研究是当前及未来研究的重点. 目前研究制备高频段负折射率材料可分为两种. 一 种是构造具有新的微纳米结构的超材料; 另一种是 利用光子晶体的电磁波禁带操控电磁波的传播, 得 到负折射率材料.

本文研究的负折射率材料选用纳米级同时具有 金属线电响应和金属开口谐振环磁响应的结构. 考 虑到其相对介电常数、相对磁导率与入射光频率之间 固有的内在联系, 有色散关系为:

1) 相对介电常数 ${ }^{[13]}$

$$
\varepsilon=1-\frac{\omega_{\mathrm{p}}^{2}}{\omega^{2}+i \gamma_{\mathrm{e}} \omega},
$$

式中 $\omega_{\mathrm{p}}$ 为材料有效等离子体频率, $\omega_{\mathrm{p}}{ }^{2}=2 \pi \mathrm{c}^{2} /\left[a_{\mathrm{e}}{ }^{2} \ln \left(a_{\mathrm{e}} / \gamma_{\mathrm{e}}\right)\right]$. 当结构单元尺寸 $a_{\mathrm{e}}$ 为纳米级别时, 可以得到 $10^{14}$ 数量 级的等离子体频率. $\gamma_{\mathrm{e}}$ 为阻尼项, 根据实验取 $\gamma_{\mathrm{e}}=$ $0.0067 \omega_{\mathrm{p}}{ }^{[13]}$.

2) 相对磁导率 [14]

$$
\mu=1-\frac{F \omega^{2}}{\omega^{2}-\omega_{0}^{2}+i \Gamma \omega},
$$

式中 $\omega_{0}$ 为材料有效谐振频率; $F$ 为每个小单元中开口 谐振环所占据的面积, $F=\pi r^{2} / a^{2}$ 确定了负磁导率的频 带宽度; $\Gamma$ 为损耗系数. 当 $\omega_{0}<\omega<\omega_{\mathrm{b}}$ 时, $\mu<0$, 式中 $\omega_{\mathrm{b}}=\omega_{0} / \sqrt{1-F}$.

本文研究选取的负折射率物性参数为: 结构单 元尺寸 $r / a=0.5$, 则有 $F=\pi r^{2} / a^{2}=0.785, \omega_{0}<\omega<2.17 \omega_{0}$. 为了简化分析计算取 $\omega_{0}=0.5 \omega_{\mathrm{p}}, \Gamma=\gamma_{\mathrm{e}}=0.0067 \omega_{\mathrm{p}}$. 为 了保证负折射率材料的相对介电常数及相对磁导率 同时为负, 取 $0.5 \omega_{\mathrm{p}}<\omega<\omega_{\mathrm{p}}$. 经过无量纲处理可以使 计算的结果适用于任何频率范围, 有 $0.5<\omega / \omega_{\mathrm{p}}<1$.

\section{3 结果与讨论}

\section{1 折射率 $\boldsymbol{n}$ 及消光系数 $\boldsymbol{\kappa}$}

材料的复折射率与相对介电常数和相对磁导率 
有如下关系:

$$
\tilde{n}=-\sqrt{\varepsilon \mu} \text {. }
$$

根据色散关系(11)和(12)式可知, 材料的折射率 $n$ 仅与 $\omega / \omega_{\mathrm{p}}$ 有关. 从图 2 中可以看出, $n$ 在 $0.5<\omega / \omega_{\mathrm{p}}<$ 0.505 范围内达到一个峰值, 此时 $|n|$ 最大. 随着 $\omega / \omega_{\mathrm{p}}$ 的增大, 折射率趋于 0 . 消光系数的变化趋势与折射 率基本一致. 当 $\omega / \omega_{\mathrm{p}}>0.667$ 时, $|n|<1$, 入射光由光密 介质射向光疏介质, 在界面处发生全反射行为. 这时 $\kappa \approx 0$. 入射波长对应的临界角满足关系 $\sin \theta_{\mathrm{c}}=n / n_{0}$. 表 1 是各入射波长对应的临界角.

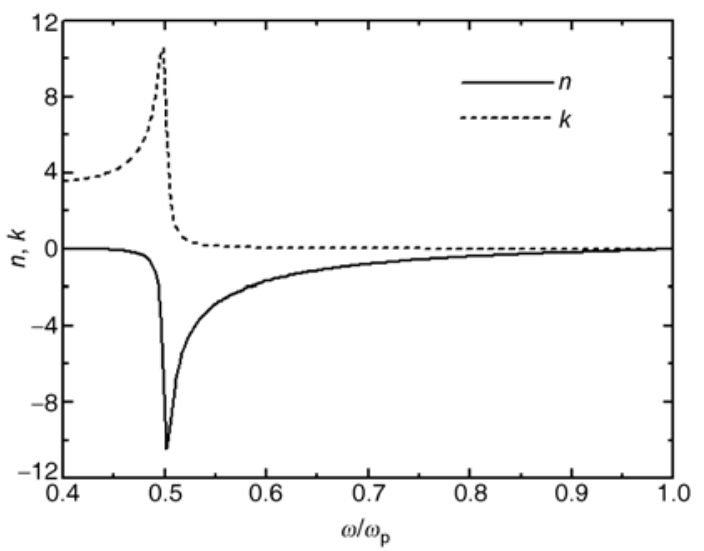

图 2 负折射率材料的折射率 $n$ 及消光系数 $\boldsymbol{k}$
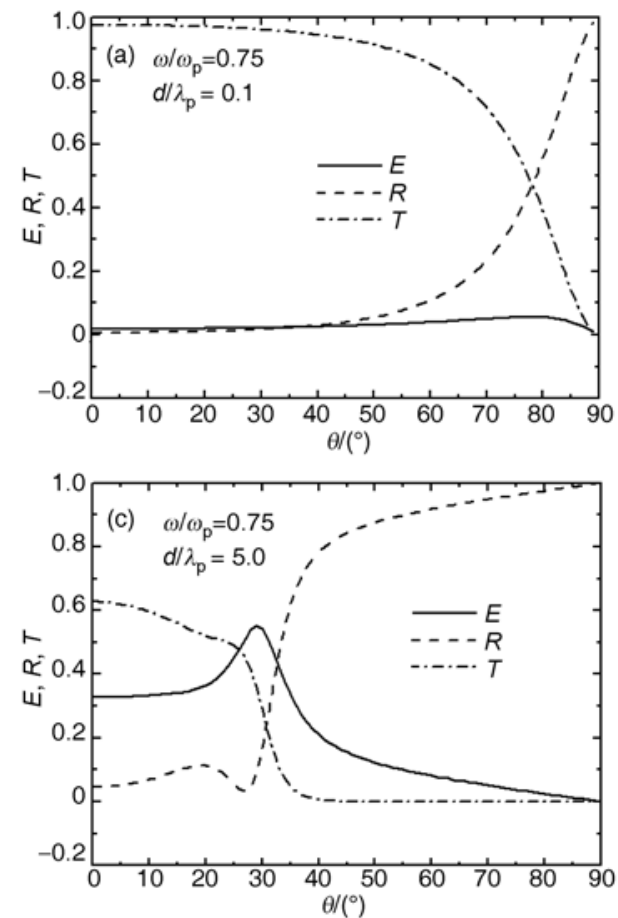

表 1 各入射波长对应临界角

\begin{tabular}{ccccccccc}
\hline$\omega / \omega_{\mathrm{p}}$ & 0.67 & 0.70 & 0.75 & 0.80 & 0.85 & 0.90 & 0.95 & 0.99 \\
\hline$n$ & -0.973 & -0.792 & -0.567 & -0.403 & -0.277 & -0.178 & -0.096 & -0.033 \\
$\theta_{\mathrm{c}}$ & $76.6^{\circ}$ & $52.3^{\circ}$ & $34.5^{\circ}$ & $23.7^{\circ}$ & $16.1^{\circ}$ & $10.3^{\circ}$ & $5.5^{\circ}$ & $1.9^{\circ}$ \\
\hline
\end{tabular}

\section{2 修逝波对薄膜偏振特性的影响}

对于正折射率材料, 热辐射从光密介质向光疏 介质传递发生全反射时，会产生修逝波. 修逝波将透 入介质约一个波长, 并沿着 $x$ 方向传播, 振幅在 $z$ 方 向按指数衰减, 如图 1 所示. 当薄膜的厚度大于 1 个 波长时, 薄膜的反射率接近 1 , 透射率接近 0 . 当热辐 射从正折射率介质(或真空)向负折射率介质传递时, 修逝波有放大效应, 将形成明显的光子隧道效应, 使 得负折射率薄膜的热辐射特性与传统材料有较大的 区别.

图 3 是 $\omega / \omega_{\mathrm{p}}$ 取 0.75 时, 不同厚度下, 薄膜的透射 率、反射率及发射率随天顶角 $\theta$ 的变化情况, 图中 $\lambda_{\mathrm{p}}$ 为 $\omega_{\mathrm{p}}$ 所对应的波长. 从表 1 可知, $\omega / \omega_{\mathrm{p}}=0.75$ 时,全反 射临界角是 $34.5^{\circ}$. 当 $d / \lambda_{\mathrm{p}}=0.1$ 和 1 时, 薄膜的透射 率和反射率随着天顶角 $\theta$ 的增大分别呈现减小和增大 的特点. 当 $d / \lambda_{\mathrm{p}}=5$ 时, 薄膜的反射率和发射率随着 天顶角的增加, 出现非单调的变化. $d / \lambda_{\mathrm{p}}=10$ 时, 上 述非单调变化现象更加明显, 薄膜的发射率和反射
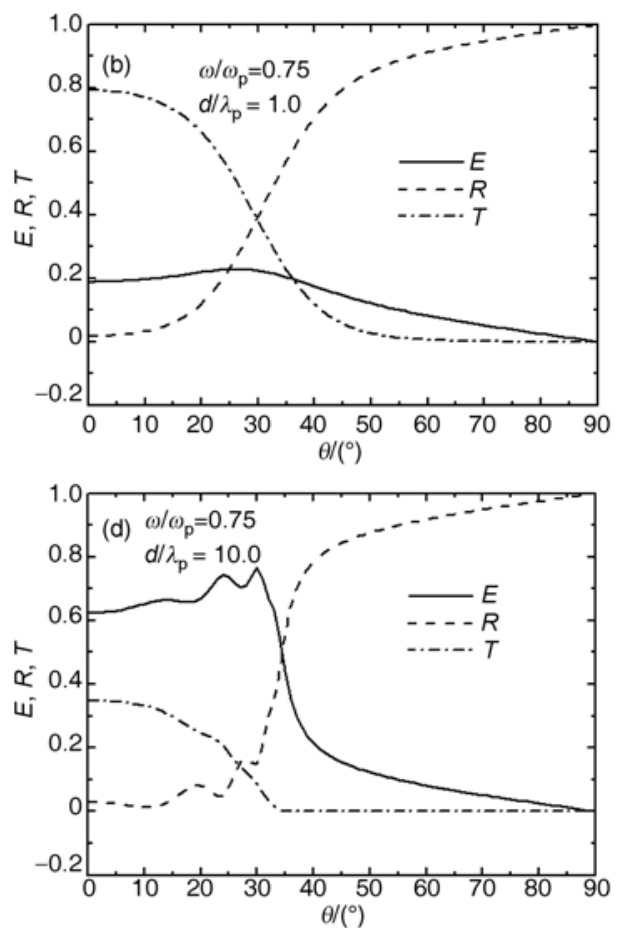

图 3 不同厚度负折射率薄膜热辐射 TM 波的方向特征 
率随天顶角增加, 出现了若干个峰值. 从计算结果可 以看出, 即使对于 $d / \lambda_{\mathrm{p}}=10$ 的情况, 天顶角大于全反 射角时不会出现反射率等于 1 的现象. 当 $d / \lambda_{\mathrm{p}}=5$, 天 顶角 $\theta=40^{\circ}$ 时, 薄膜的透射率约为 0.01 . 这说明负折 射率介质中, 由于修逝波有放大作用. 当薄膜的厚度 超过入射波长, 也能形成光子隧道. 负折射率薄膜的 热辐射特性是干涉效应 ${ }^{[15]}$ 和光子隧道效应共同作用 的结果.

\section{3 薄膜热发射的方向特性}

常规尺度下, 采用几何光学研究半透明材料的 表观发射率时, 发现其呈现准各向同性的特点, 法线 方向的发射率较大, 在接近 $90^{\circ}$ 方向很小. 对于薄膜 材料, 考虑波动光学效应, 法线方向通常不是发射率 的峰值方向, 并且有时热发射率的峰值远离法线方 向, 并且有些情况下比法线方向的发射率大很多.

图 4 是无量纲角频率 $\omega / \omega_{\mathrm{p}}$ 取不同值时, 薄膜热发 射率的计算结果. 可以看出, 随着 $\omega / \omega_{\mathrm{p}}$ 值的增大, 发 射率的峰值对应角度逐渐减小. 通过改变薄膜结构 和物性参数, 可以对负折射率薄膜热辐射的发射方 向特性进行调制.
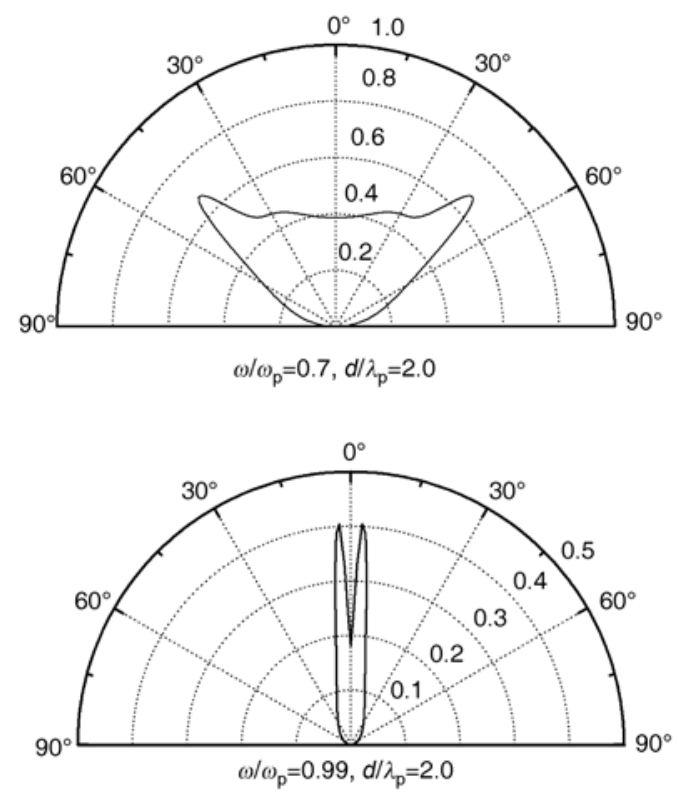

图 4 不同 $\omega \omega_{\mathrm{p}}$ 时薄膜的热发射率

\section{4 薄膜热辐射的光谱特性}

图 5 是不同角度时, 3 种不同厚度的负折射率薄 膜发射率随 $\omega / \omega_{\mathrm{p}}$ 的变化情况. 从计算结果可以看到, 发射角 $\theta=70^{\circ}$ 、薄膜厚度为 $\lambda_{\mathrm{p}}$ 时, 随着 $\omega / \omega_{\mathrm{p}}$ 的变化,
发射率值存在较为明显的震荡, 出现明显的干涉现 象. 而薄膜厚度在取 $d / \lambda_{\mathrm{p}}=0.1$ 及 $d / \lambda_{\mathrm{p}}=10$ 时, 都没有 干涉现象. 发射角 $\theta=30^{\circ}, d / \lambda_{\mathrm{p}}=1$ 时, 出现不明显的 相干现象. 这是因为, 随着热发射角度的增大, 进入 电磁波在薄膜上、下表面间经历的路程较长, 不同相 位波向前传递的数目增多, 产生干涉的现象明显. 在 热发射角相同情况下, 由于消光系数的影响, 薄膜厚 度的增加, 使得电磁波传播经历的衰减增强, 不容易 形成相位不同的波向前传递，干涉现象不容易发生. 对于同样的发射角和薄膜厚度, 全反射现象在 $\omega / \omega_{\mathrm{p}}>0.7$ 时更加明显, 薄膜的吸收率减少, 而使在 $0.7<\omega / \omega_{\mathrm{p}}<1$ 范围内, 观测不到干涉现象. 结果表明, 通过改变薄膜结构和物性参数, 可以对负折射率薄 膜热辐射的光谱特性进行调制, 研制出具有特定发 射率峰值的负折射率薄膜.
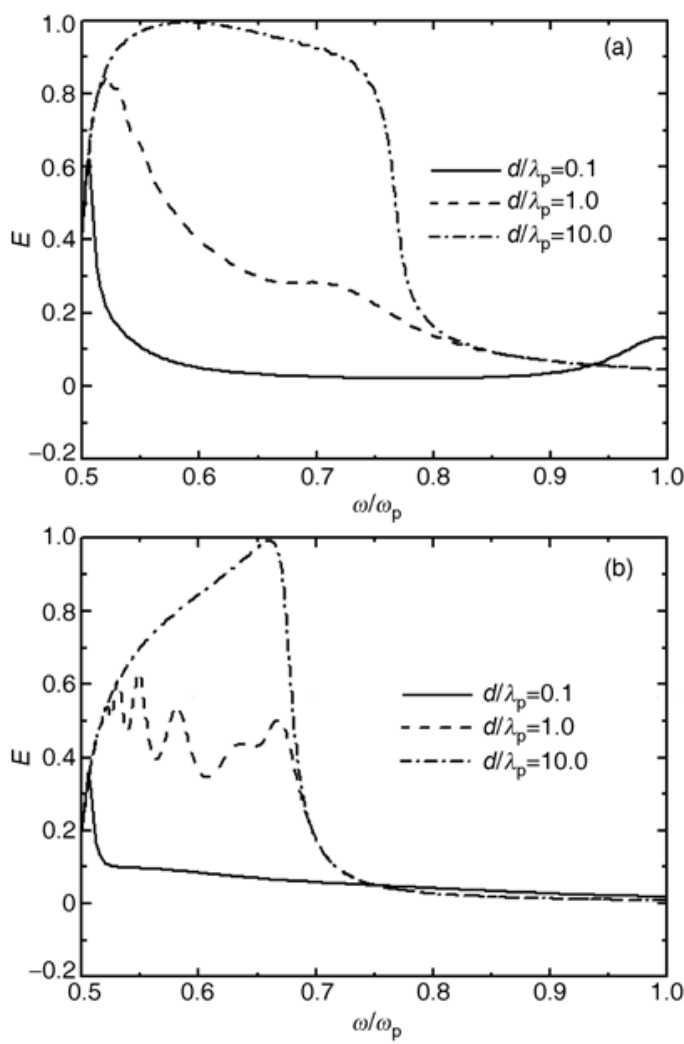

图 5 不同角频率下发射率的特征

(a) $\theta=30^{\circ}$, (b) $\theta=70^{\circ}$

\section{4 结论}

本文利用传输矩阵方法及基尔霍夫定律推导了 $\mathrm{TM}$ 偏振波的偏振特性的计算公式, 研究 TM 波特性 时考虑了负折射率材料相对介电常数及相对磁导率 
与入射光频率之间存在的固有联系. 分析了修逝波 对 TM 偏振波偏振特性的影响.

负折射率薄膜的热辐射特性是由两个作用共同 影响所产生的结果, 一个作用是薄膜结构对电磁波
的干涉效应，另一个作用是负折射率材料对修逝波 的放大导致的光子隧道效应. 研究结果表明, 通过调 整薄膜结构的体系参数, 可以对其热辐射的方向特 征和光谱特征进行相应的调制.

\section{参考文献}

1 Veselago V G. The electrodynamics of substances with simultaneously negative values of $\varepsilon$ and $\mu$. Sov Phys Usp, 1968, 10: 509-514

2 Shelby R A, Smith D R, Schutz S. Experimental verification of a negative index of refraction. Science, 2001, 292(5514): 77-79 [DOI]

3 Grigorenko A N, Geim A K, Gleeson H F, et al. Nanofabricated media with negative permeability at visible frequencies. Nature, 2005, 438(7066): 335-338 [DOI]

4 Shalaev V M, Cai W S, Chettiar U K, et al. Negative index of refraction in optical metamaterials. Opt Lett, 2005, 30(24): 33563358 [DOI]

5 曹云建，文光俊，吴凯敏，等。一种新型负折射率微波媒质的设计与仿真模拟．科学通报, 2006, 51(22): 2612-2617

6 Pendry J B. Negative refraction makes a perfect lens. Phys Rev Lett, 2000, 85: 3966 - 3969 [DOI]

7 Grigorenko A N, Geim A K, Gleeson H F, et al. Nanofabricated media with negative permeability at visible frequencies. Nature, 2005, 438(7066): 335-338[DOI]

8 Valentine J, Zhang S, Zentgraf T, et al. Three-dimensional optical metamaterial with a negative refractive index. Nature, 2008, 455(7211): 376-379[DOI]

9 Yao J, Liu Z W, Liu Y M, et al. Optical negative refraction in bulk metamaterials of nanowires. Science, 2008, 321(5891): 930930 [DOI]

10 Cho A. Physics: Voilà! Cloak of invisibility unveiled. Science, 2006, 314(5798): 403 - 403[DOI]

11 Yeh P. Optical Waves in Layered Media. New York: Wiley, 1988

12 Zhang Z M, Fu C J, Zhu Q Z. Optical and thermal radiative properties of semiconductors related to micro/nanotechnology. In: Hartnett J, Cho Y, Greene G, eds. Advances in Heat Transfer 37. St. Louis: Academic Press, 2003

13 Pendry J B, Holden A J, Stewart W J, et al. Extremely low frequency plasmons in metallic mesostructures. Phys Rev Lett, 1996, 76: 4773-4776[DOI]

14 Pendry J B, Holden A J, Robbins D J, et al. Magnetism from conductors and enhanced nonlinear phenomena. IEEE T Microw Theory, 1999, 47: 2075-2084[DOI]

15 韩茂华, 梁新刚, 黄勇. SiC 薄膜的热辐射特性研究. 科学通报, 2005, 50(6): 588-591 